\title{
Pattern Formation and Spatiotemporal Irregularity in a Model for Macrophage-Tumour Interactions
}

\author{
Markus R. Owen* and Jonathan A. Sherratt \\ Nonlinear Systems Laboratory, Mathematics Institute, University of Warwick, Coventry \\ CV4 7AL, U.K.
}

(Received on 17 February 1997, Accepted in revised form on 10 June 1997)

\begin{abstract}
Solid tumours do not develop as a homogeneous mass of mutant cells, rather, they grow in tandem with normal tissue cells initially present, and may also recruit other cell types including lymphatic and endothelial cells. Many solid tumours contain a high proportion of macrophages, a type of white blood cell which can have a variety of effects upon the tumour, leading to a delicate balance between growth promotion and inhibition. In this paper we present a brief review of the main properties and interactions of such tumour-associated macrophages, leading to a description of a mathematical model for the spatial interactions of macrophages, tumour cells and normal tissue cells, focusing on the ability of macrophages to kill mutant cells. Analysis of the homogeneous steady states shows that, for this model, normal tissue is unstable to the introduction of mutant cells despite such an immune response, but that the composition of the resulting tumour can be significantly altered. Including random cell movement and chemical diffusion, we demonstrate the existence of travelling wave solutions connecting the normal tissue and tumour steady states, corresponding to a growing tumour, and of the development of a spatial instability behind the wave front. Numerical solutions are illustrated in one and two dimensions. We go on to estimate macrophage motility parameters using data from Boyden chamber experiments. We then extend our model to include macrophage chemotaxis, that is, their directed movement in response to gradients of chemicals secreted by tumours. Solutions in one dimension indicate the possibility of spatiotemporal irregularities within the growing tumour, which are deduced to be the result of a series of bifurcations as the effective domain length increases, leading to a permanently transient solution. These results suggest that tumour heterogeneity may arise, in part, as a natural consequence of the macrophage infiltration. Recent experiments suggest that macrophages may indeed be involved in spatiotemporal variations within some human tumours.
\end{abstract}

(C) 1997 Academic Press Limited

\section{Introduction}

It has been known for many years that most solid cancers are heterogeneous, both spatially and in terms of their populations of different cell types. Whilst malignant cells drive the growth of a tumour, normal tissue cells can continue to proliferate, and the additional recruitment from other sites of a variety of cell types - including lymphocytes, macrophages, and endothelial cells - is well documented. Macrophages

\footnotetext{
* Author to whom correspondence should be addressed. Present address: Dept of Mathematics, University of Utah, Salt Lake City, UT 84112, USA. E-mail: owen@math.utah.edu

are the mature form in tissue of a type of white blood cell known as a monocyte. They form an important arm of the immune system, with the ability to recognise and phagocytose damaged cells, cellular debris and foreign objects. In addition, they play an important role in the response to injury, infection, and inflammation. In the context of the tumour infiltrate described above, they are referred to as tumour associated macrophages (TAM). TAM often form a significant proportion of the total mass of a tumour, over $50 \%$ in many cases of breast carcinoma (O’Sullivan \& Lewis, 1994), and can affect tumour angiogenesis, growth rate, connective tissue formation and dissolution, and the killing of malignant

C) 1997 Academic Press Limited 
cells. Tumour cells and TAM both release factors which can affect each others activity, and the details of this regulation can have important consequences for the survival of tumours, and for the possibility of manipulating the balance between pro- and anti-tumour effects to benefit patients. For detailed reviews of these interactions and their mechanisms see papers by Esgro et al. (1990), Hamilton \& Adams (1987), Mantovani (1989, 1990), and Mantovani et al. (1992). It is important to stress that macrophages form only one part of a complex interaction between tumours and the immune system, which can be either stimulatory or inhibitory for tumour growth (Prehn, 1994).

Previously we used an ordinary differential equation model for the early growth of tumours to investigate the TAM-tumour cell interaction (Owen \& Sherratt, 1997), and in this paper we extend that work to study the consequences for the spatial structure of the tumour. We will concentrate on the inhibitory effects of macrophages on the growth of an avascular tumour - that is, a tumour small enough for its nutritional needs to be satisfied by diffusion from nearby blood vessels.

A number of approaches have been used to model different aspects of such growth. Michelson \& Leith (1991), Sherratt \& Nowak (1992), and Wheldon (1975) concentrated on the regulation by growth factors of normal and mutant cell growth. Multicell spheroids are an in vitro analogue for avascular tumour growth, which have been extensively studied using a variety of approaches including reaction diffusion models (Greenspan, 1972) and the application of nonlinear elasticity theory (Chaplain \& Sleeman, 1993). Markovitch (1993) and Tomlinson \& Bodmer (1995) considered the role of apoptosis in tumour growth, an important feature which has been somewhat neglected in the development of tumour growth models. Perumpanani et al. (1996) focussed on interactions between malignant, benign, and normal cells, and the extracellular matrix. Yakovlev (1996) used a stochastic framework to investigate tumour latency.

A variety of detailed models of tumour immunology have also been proposed-see Adam \& Bellomo (1997) for a review. The influence of growth factors and modulators on the immune response to tumour growth was considered by Adam (1993), and Albert et al. (1980). Kuznetsov et al. (1993) focussed on the interactions between different immune cell types, and on the dynamics of immunogenic tumours (Kuznetsov et al., 1994). A number of authors, including Lefever et al. (1992), Perelson \& Bell (1982), and Perelson et al. (1984), have concentrated on the details at the cellular level of immune cell binding and the delivery of lethal hits.

The mechanisms we consider here are cell proliferation, aggregation of macrophages at the tumour site, and their killing of mutant cells. Macrophages in normal tissue do not proliferate significantly, but at least in some cases TAM differ in this respect due to the production by tumour cells of regulators such as macrophage colony stimulating factor (M-CSF), and enhanced expression of receptors for M-CSF on the surface of TAM (Bottazzi et al., 1990). This evidence is restricted to a few mouse sarcoma cell lines, and in humans M-CSF seems to promote macrophage survival, but is not a good proliferative stimulus (Mantovani et al., 1992).

The basic mechanism by which macrophages are recruited to a tumour site is chemotaxis. Monocytes circulate in the bloodstream, adhering to capillary walls where the concentration of chemotactic chemical is sufficiently high, and enter the tissue, where they mature into macrophages. Along with macrophages already resident, they move up chemical gradients towards the tumour. There is a large body of experimental work on the production by mutant cells of macrophage chemoattractants. Results include the identification of monocyte chemotactic protein (MCP-1) (Bottazzi et al., 1983), followed more recently by the related proteins MCP-2 and MCP-3 (van Damme et al., 1992), and other chemoattractants including M-CSF (Wang et al., 1987, 1988). An important correlation has repeatedly been found between the extent of chemotactic activity, as determined by in vitro assays or imposed by genetic alteration, and the proportion of TAM within tumours in vivo (Bottazzi et al., 1983, 1985, 1992; Walter et al., 1991).

Finally, macrophages are able to lyse tumour cells in preference to normal cells. This is dependent on biochemical activation (Mantovani, 1990). A two stage activation process has been postulated, in which newly recruited macrophages are first "primed" by interferon- $\gamma$, and subsequently activated to full competence (Hamilton \& Adams, 1987). Other cytokines such as tumour necrosis factor and M-CSF have been shown to induce or augment macrophage activation (Mace et al., 1988; Mantovani, 1990; Sampson-Johannes \& Carlino, 1988), and recent evidence suggests that cytokine-independent pathways may also be involved (Keller, 1993). Some of these macrophage-activating factors are produced by certain tumour cell lines (Mantovani, 1990; Mantovani et al., 1992). Esgro et al. (1990) note that once macrophages have been activated and then lysed a 
tumour cell, they revert to an inactivated state ready for reactivation.

Once activated, the first step in tumour cell killing is the formation of a macrophage-tumour cell complex. This direct cell-cell contact is a key part of the lysis (Hamilton \& Adams, 1987; Jonjic et al., 1992), and may also upregulate macrophage activation (Mantovani, 1993). Many different mechanisms have been proposed for the actual lysis process within the complex, all involving secretion of cytolytic factors. In particular, a nitrogen monoxide mediated mechanism has been determined in detail (Hibbs, 1991). A detailed review of cytolytic mechanisms is given by Hamilton \& Adams (1987).

Given this information, we describe in the next section a model in which mutant cells, with a proliferative advantage over normal tissue cells, produce a generic chemical which regulates macrophage proliferation, influx, activation, and complex formation. Subsequently we give the details of the homogeneous steady states and their stability, and then focus on model solutions with only random motion of macrophages, i.e. without chemotaxis. In Section 4, we demonstrate the existence of travelling wave solutions connecting the normal tissue steady state ahead of the wave with a tumour steady state behind. The steady state behind the wave is shown in Section 5 to become unstable to spatial perturbations as the diffusion coefficient of a chemical regulator increases through a critical value, and solutions in two dimensions are illustrated in Section 6. Before including macrophage chemotaxis, the macrophage motility parameters are estimated from experimental data using a mathematical model for the experimental procedure, as discussed in Section 7. Finally, in Section 8 we describe the results of simulations in one dimension, including chemotaxis using these parameter estimates. In particular we note the existence of spatiotemporally irregular wakes behind the wave front.

\section{Modelling}

As variables for our model we use population densities of macrophages, $l(x, t)$; mutant cells, $m(x, t)$; normal cells, $n(x, t)$; and macrophage-mutant-cell complexes, $c(x, t)$. It is important to note that the macrophage population is highly heterogeneous in terms of individual macrophage susceptibility to activation and chemotaxis (Adams \& Hamilton, 1984), and so one could envisage a much more extensive set of variables which would include various macrophage subpopulations. We feel that it is appropriate to consider the simpler case first, whilst bearing this additional complexity in mind, with a view to further development of the model. We also make the simplifying assumption that there is a single chemical regulator, of concentration $f(x, t)$, responsible for macrophage activation, proliferative control, and stimulating their influx from the bloodstream. This is a reasonable simplification, since the various regulators involved all derive primarily from mutant cells.

Quiescent macrophages must be biochemically activated before they form a complex with, and then lyse, a mutant cell, as described above. We assume that complex formation is the rate limiting step, and hence that its rate is linear with respect to the concentration of the activating chemical, and the macrophage and mutant cell densities. In addition, we assume that the complex returns viable macrophages after lysis of the mutant cell (Esgro et al., 1990). Schematically this can be represented in the following form:

$$
f+l+m \stackrel{k_{1}}{\rightarrow} c+f \quad c \stackrel{k_{2}}{\rightarrow} l+\text { debris. }
$$

Here $k_{1}$ and $k_{2}$ are positive constants. We must stress that there is no definitive experimental data on the details of this tumour cell destruction; we have considered some alternative formulations, such as separation of activation and complex formation, and degradation of the chemical, and preliminary results suggest that these changes do not alter the qualitative features of the model kinetics.

The remaining assumptions that we make with regard to macrophages are: (i) they proliferate only in the presence of the chemical regulator (Bottazzi et al., 1990) and that such proliferation increases linearly with concentration; (ii) proliferation is limited by the crowding effect of all cell types; (iii) there is an influx from capillaries, which increases linearly with regulator concentration; (iv) they move chemotactically up gradients in regulator concentration; and (v) they die with some constant rate per cell. In the absence of chemical regulator, the normal background level of tissue macrophages is maintained by a constant influx, at a rate denoted by $I$. Combining these terms gives the following conservation equation for macrophages:

$$
\begin{gathered}
\overbrace{\frac{\partial l}{\partial t}=D_{l} \nabla^{2} l-\chi_{l} \nabla(l \nabla f)}^{\text {cell migration }}+\overbrace{\frac{\alpha f l\left(N+N_{e}\right)}{N+l+m+n}}^{\text {proliferation }} \\
+\overbrace{I(1+\sigma f)-}^{\text {influx }} \overbrace{k_{1} f l m+k_{2} c-\overbrace{\delta_{l} l} l}^{\text {lysis death }}
\end{gathered}
$$


Most tumours arise initially from a mutation that affects the control of cell division. This can be due to the expression of an oncogene or the loss of a tumour suppressor gene (Fearon \& Vogelstein, 1990; Volpe, 1990; Weinberg, 1989); in either case the result is a proliferative advantage for the mutant cell over its peers. Previous mathematical models have focused on the different types of mutation and their consequences (Sherratt \& Nowak, 1992; Wheldon, 1975). Here our focus is on the role of macrophages during the early stages of tumour growth, and thus we use a simplification in which the dynamics of mutant and normal cells are alike except for the removal of mutant cells by macrophages, and a scaling of the mutant cell proliferation rate, compared to their normal counterparts, of $\xi>1$. We use the same crowding term as discussed above, and a growth rate in normal tissue of $\delta$, balanced by an equal rate of cell death, so that the conservation equations for mutant and normal cells are:

$$
\begin{aligned}
& \frac{\partial m}{\partial t}=\overbrace{D_{m} \nabla^{2} m}^{\text {cell migration }}+\overbrace{\frac{\xi \delta m\left(N+N_{e}\right)}{N+l+m+n}}^{\text {proliferation }} \overbrace{-\delta m}^{\text {death }} \overbrace{-k_{1} f m}^{\text {lysis }} \\
& \frac{\partial n}{\partial t}=D_{n} \nabla^{2} n+\frac{\delta n\left(N+N_{e}\right)}{N+l+m+n}-\delta n
\end{aligned}
$$

The sole source of generic chemical regulator is mutant cells, and we assume a constant secretion rate $\beta$ per unit of mutant cell density, and a linear natural decay with rate $\delta_{f}$. Complex density is also assumed to decay linearly, with rate $\delta_{c}$. Including the binding and lysis terms discussed above completes the derivation of our model:

$$
\begin{aligned}
& \frac{\partial f}{\partial t}=\overbrace{D_{f} \nabla^{2} f}^{\text {diffusion }} \overbrace{+\beta m}^{\text {prod }^{n}} \overbrace{-\delta_{f} f}^{\text {decay }} \\
& \frac{\partial c}{\partial t}=\overbrace{D_{c} \nabla^{2} c}^{\text {migration }}+\overbrace{k_{1} f m-k_{2} c}^{\text {lysis }}-\overbrace{\delta_{c} c . \quad \text { death }}^{\text {leth }}
\end{aligned}
$$

The model given by eqns $(1-3)$ is non-dimensionalized using rescalings that are described in the Appendix.

There is insufficient experimental data available to determine all the model parameters for any particular tumour type. We list in the Appendix typical order of magnitude estimates for the parameters, with brief justifications. All the phenomena described in this paper occur for ranges of parameters within these estimates.

\section{Homogeneous Steady States and Stability}

In this section we summarise briefly the homogeneous steady states of the dimensionless model (A.1), and their stability to homogeneous perturbations.

There are four types of steady state to consider. The first two are simple to analyse, a macrophages-only state

$$
l=\frac{I}{\delta_{l}}, \quad m=n=f=c=0,
$$

and normal tissue consisting of normal cells and macrophages:

$$
l=\frac{I}{\delta_{l}}, \quad m=0, \quad n=1-\frac{I}{\delta_{l}}, \quad f=c=0 .
$$

Straightforward linear stability analysis shows that both these steady states are unstable to the introduction of mutant cells.

The third type is that of a tumour with no normal cells remaining, denoted the mutant-only steady state, and the fourth steady state is of mixed type, with all cell species coexisting. Real tumours certainly contain a variety of cell types (Mantovani, 1990), and other models (Sherratt \& Nowak, 1992; Wheldon, 1975) also predict this type of solution. If the growth advantage of mutant cells is too high, coexistence is not possible, and steady states of this type cannot exist. Figure 1 illustrates the way in which these last

(a) (b)

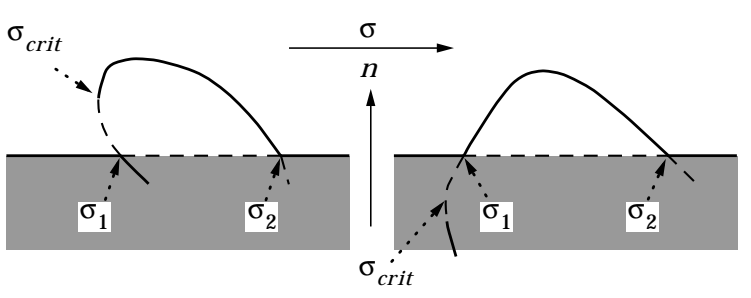

FIG. 1. Schematic representation of the bifurcation structures for the homogeneous tumour steady states of the dimensionless model (A.1). Solid lines indicate stability, dashed lines instability. The bifurcation parameter $\sigma$ is the measure of the increase in macrophage influx from the bloodstream in response to chemoattractants secreted by tumour cells. Tumour stady states of the model (A.1) are of two types: (i) mutant-only $(n=0)$, where no normal cells suvive, indicated by the horizontal lines in the diagram; (ii) coexistance $(n \neq 0)$, where normal cells continue to proliferate along with mutant cells, indicated by the curve. We do not consider coexistence steady states with negative cell densities, corresponding to those in the shaded area $n<0$. There are two types of bifurcation structure, which depend upon the nature of the coexistence steady state at $\sigma=\sigma_{\text {crit }}$ : (a) $\left.n\right|_{\sigma=\sigma_{\text {crit }}}>0$, (b) $\left.n\right|_{\sigma=\sigma_{\text {crit }}}<0$. 
two steady states change with parameters. A number of bifurcation points have been identified, where the number and stability of steady states changes. This is discussed in some detail in Owen \& Sherratt (1997); for the purposes of this paper the key result is that, with respect to homogeneous perturbations, at least one tumour steady state is stable, and that for realistic parameter ranges there is only a very small region of parameter space in which both coexistence and mutant-only steady states are stable. The bifurcations described can be found by varying a number of parameters, but for convenience we fix parameter sets except for $\sigma$, the macrophage influx response parameter, which can be varied to give the full spectrum of possible behaviours.

\section{Travelling Wave Solutions}

Numerical simulations of (A.1) show that a localised mutation in normal tissue induces a travelling wave solution originating from the site of mutation, with either the coexistence or mutant-only
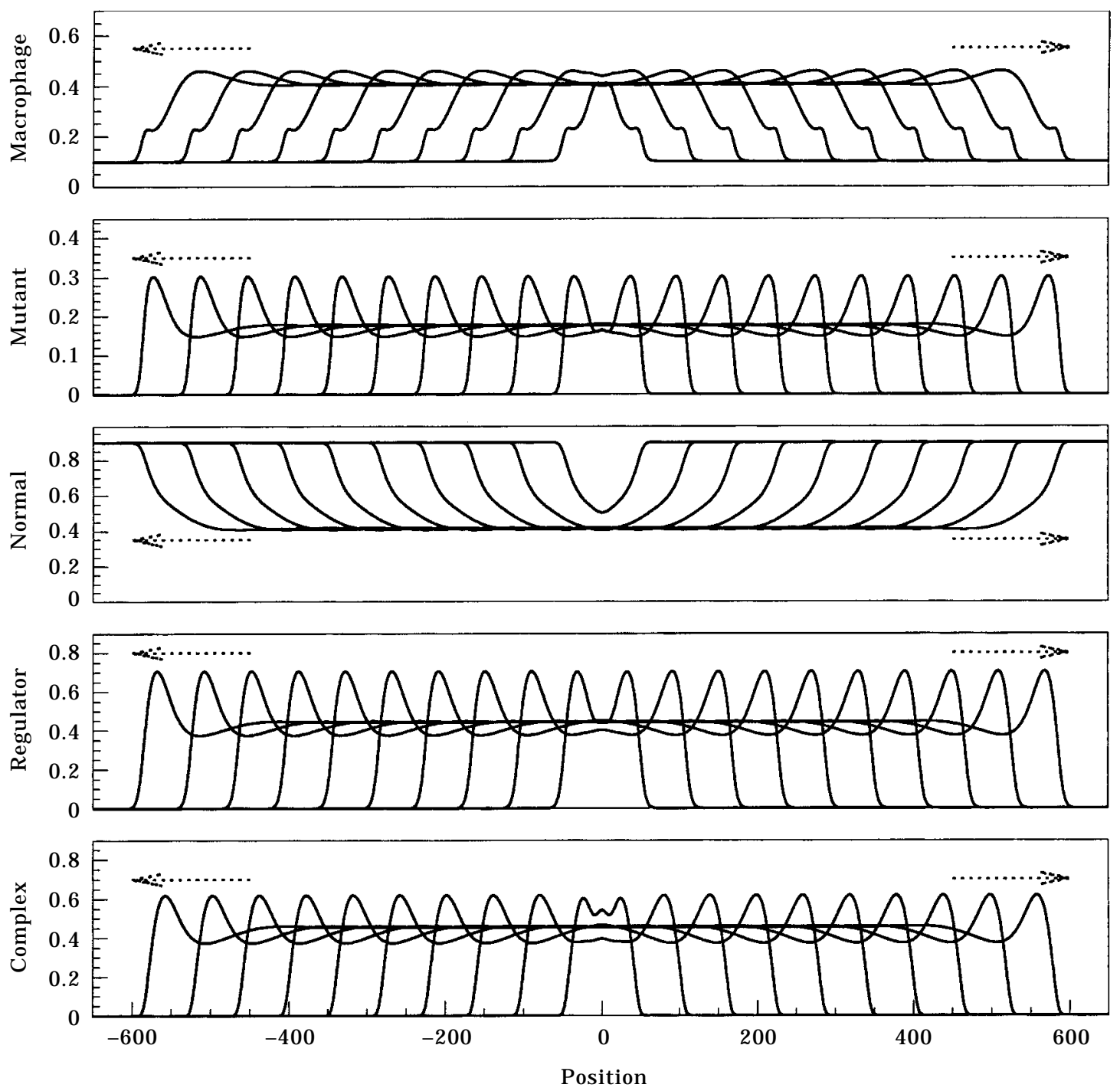

FIG. 2. Numerical simulation in one dimension of the dimensionless model (A.1), with no macrophage chemotaxis $\left(\chi_{l}=0\right)$, showing the evolution from a localized mutation at the centre. The initial conditions were the normal tissue steady state $l=0.1, m=0, n=0.9, f=0$, $c=0$ on the whole domain, apart from the mutation $l=0, m=1, n=0, f=0, c=0$ for $-0.5<x<0.5$. A travelling wave is established as the tumour grows, with the composition behind the wave front that of the homogenous stable steady state, which is dependent on the kinetic parameters. In this case the coexistence steady state is stable. The solution is plotted at intervals of 10 dimensionless time units, up to a final time of 100 , at which point the numerically calculated wave speed $=6.00738$, compared to the predicted speed of 6 , a difference of less than $0.005 \%$. This corresponds to a dimensional speed of $1.2 \times 10^{-10} \mathrm{~ms}^{-1} \approx 0.01 \mathrm{~mm}$ day $^{-1}$. The parameters are: $D_{l}=D_{m}=D_{n}=5$, $D_{f}=30, D_{c}=2.5, \alpha=0.01, \beta=5.0, \delta_{c}=0.5, \delta_{f}=2.0, \delta l=0.1, N=1, I=0.01, k_{1}=10.0, k_{2}=0.2, \sigma=58, \xi=2.8$. 
tumour steady state behind the wave, depending on the kinetic parameters. Figure 2 illustrates such a simulation, with parameters such that the stable homogeneous steady state, and hence the state behind the wave, is of coexistence type.

It is usual to investigate travelling wave solutions by introducing $z=x-$ at as the new independent variable, where $a$ is the speed of the wave. This substitution transforms the five partial differential equations into a system of ten first order ordinary differential equations. Experience with other reactiondiffusion systems suggests that, ahead of the wave, the solution will decay to the normal tissue steady state at a rate determined by the eigenvalue with the least negative real part; i.e. $\lambda_{m}=$ $\left(-a+\sqrt{a^{2}-4 D_{m}(\xi-1)}\right) /\left(2 D_{m}\right)$. Because biologically realistic solutions require $l, m, n, f$ and $c$ to be non-negative, this eigenvalue must be purely real, so that $a \geqslant 2 \sqrt{D_{m}(\xi-1)}$. By analogy with the Fisher equation, we expect a localised initial tumour cell population to evolve to a travelling wave with the minimum wavespeed $a=2 \sqrt{D_{m}(\xi-1)}$, and extensive numerical simulations support this. Note that the predicted wavespeed is dependent only on the growth advantage and random motility of mutant cells, and not on the immune response.
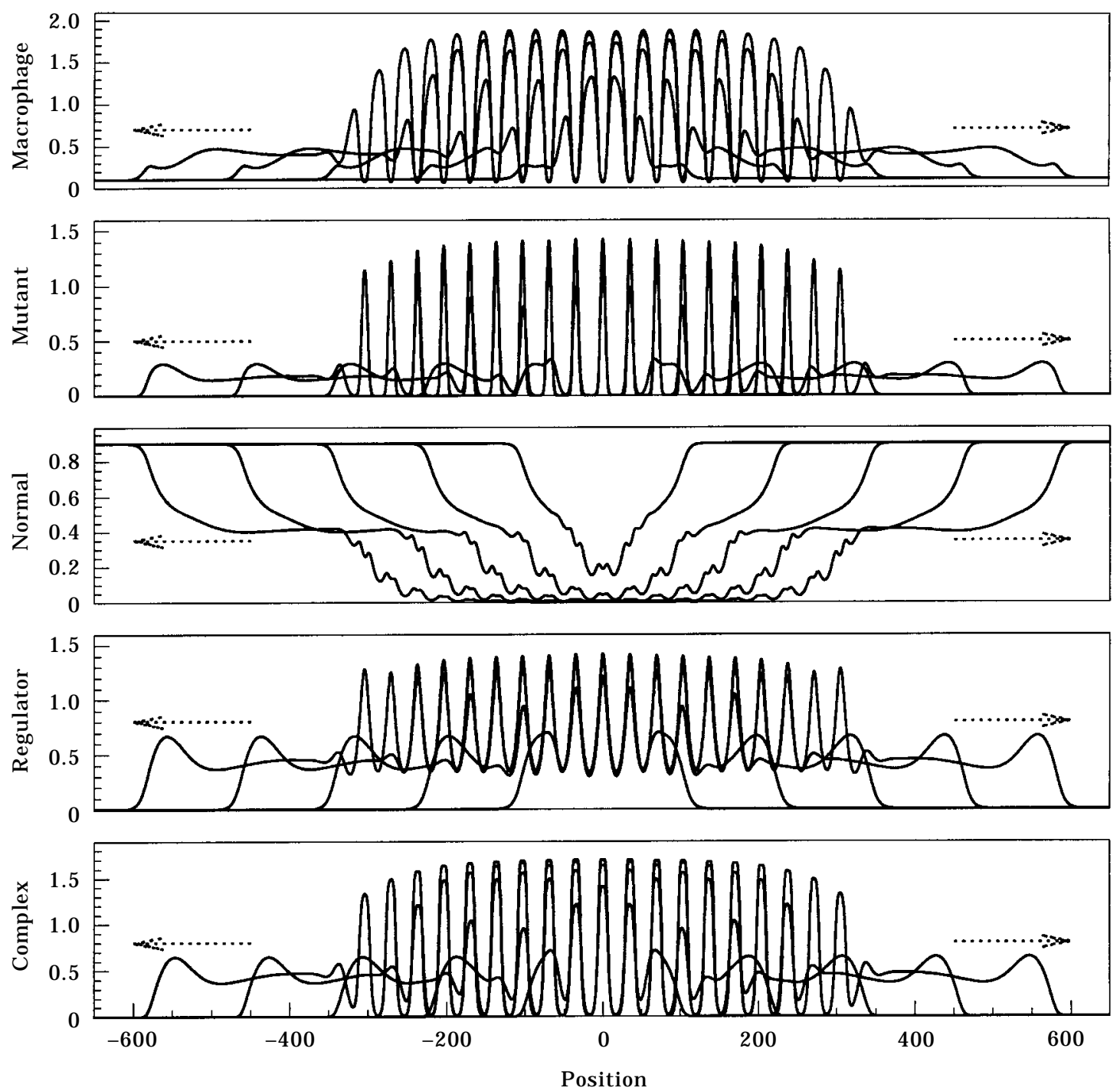

FIG. 3. Numerical simulation of the dimensionless model (A.1) in one dimension, showing the evolution of a travelling wave from a localized mutation at the centre. The initial conditions were as in Fig. 2. A stationary spatial pattern forms behind the leading wave front, whose wavelength is approximately 33 dimensionless units, i.e. 33 typical cell lengths. The wave front remains well defined, with a wave speed of 6.00436 , within $0.005 \%$ of the predicted speed. The parameters are: $D_{l}=D_{m}=D_{n}=5, D_{f}=100, D_{c}=2.5, \alpha=0.01, \beta=5.0$, $\delta_{c}=0.5, \delta_{f}=2.0, \delta_{l}=0.1, N=1, I=0.01, k_{1}=10.0, k_{2}=0.2, \sigma=58, \xi=2.8$. 


\section{Spatial Pattern Formation}

Our calculations of the wavespeed, combined with the evidence of numerical simulations, shows that the immune response has no effect on the rate of tumour growth, but that the parameters of the temporal kinetics have a significant effect on the tumour composition behind the wave front. In this section we will show that more complicated solutions are possible, in which the steady state behind the advancing tumour front becomes unstable to spatial perturbations. Figure 3 illustrates such a solution. Note that the pattern is stationary behind the wave, growing in extent but not changing in form or location. Such regular patterns are of course not observed in the stochastic, inhomogeneous environment of a real tumour, but their observation in model solutions suggests that the macrophage-tumour interaction may be a first step towards the more irregular spatial inhomogeneities seen in the real situation.

This pattern formation is an example of a Turing mechanism, in which the mutant cells are the local activator, and the chemical regulator is the long-range inhibitor. Thus, an intuitive explanation for this spatial instability, illustrated in Fig. 4, is that given a local perturbation increasing the density of mutant cells, chemical regulator production will also increase locally. Then if the chemical diffuses fast enough, it will act non-locally to activate macrophages to the tumouricidal state and to stimulate an additional influx of macrophages. At the same time macrophage

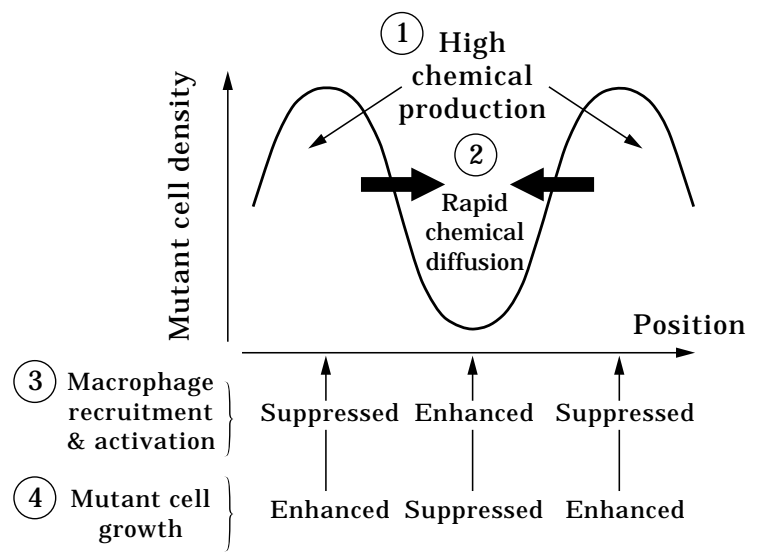

FIG. 4. An intuitive explanation for the development of spatial inhomogeneities within a tumour, due to interactions with macrophages. A locally elevated mutant cell density is reinforced by the consequently elevated chemical regulator production (1) - if the chemical diffuses sufficiently fast (2), then local macrophage recruitment and activation will be supressed (3), and hence mutant cell growth enhanced (4). Correspondingly, non-local recruitment and activation of macrophages will be enhanced, and mutant cell growth supressed. activation will be locally suppressed, so that the local mutant cell growth will effectively be enhanced, and the original perturbation will grow in time.

The first step to an analysis of the patterning phenomenon is to linearize the model (A.1) about the stable homogeneous steady state. A general perturbation can then be expressed as a linear combination of spatial modes; mathematically, we substitute solutions proportional to $e^{i \kappa x+\lambda t}$ into the linearized equations and look for non-trivial solutions, giving the dispersion relation for the real part of $\lambda$ (i.e. the growth rate) as a function of wavenumber $\kappa$. The homogeneous steady state is then stable to all spatial perturbations if $\operatorname{Re}(\lambda)<0$ for all $\kappa \in \mathrm{R}$, while if $\operatorname{Re}(\lambda)>0$ for some $\kappa$ then the mode corresponding to that wavenumber will grow exponentially in time, destabilising the homogeneous steady state.

Figure 5 illustrates the numerically calculated dispersion relation for a parameter set for which a range of wavenumbers are unstable, and Fig. 3 shows the corresponding numerical simulation of the model equations. Although the wave front is moving, the pattern which grows behind the front is stationary in space, with a wavelength of 33 cell lengths ( $\approx 0.33 \mathrm{~mm}$ ). The wavenumber of the fastest growing

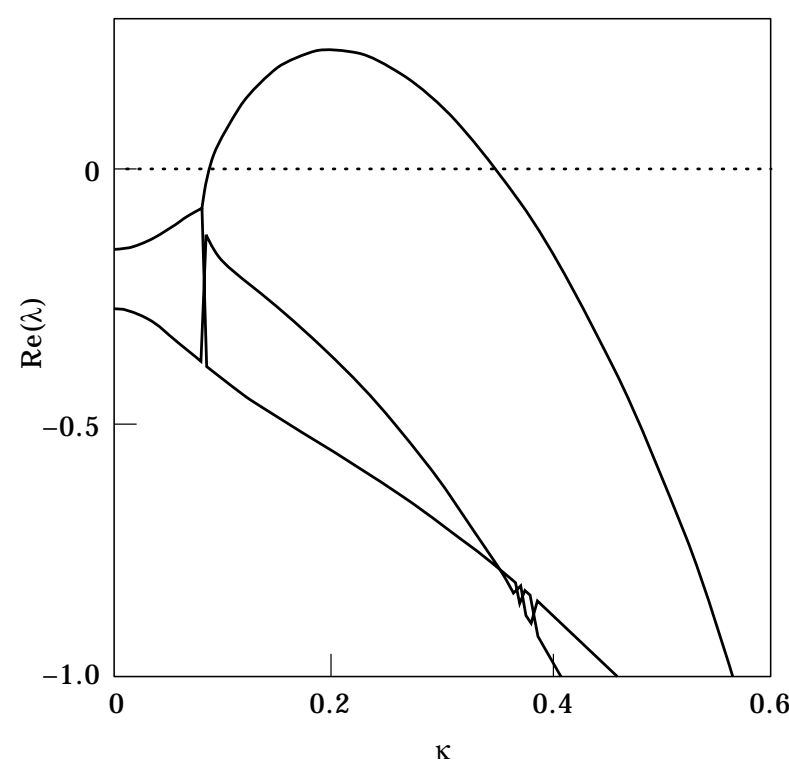

FIG. 5. Numerically calculated dispersion relation for the model (A.1), showing $\operatorname{Re}(\lambda)$ as the wavenumber $\kappa$ varies. The different curves are for the different eigenvalues. The fastest growing mode is approximately $\kappa=0.2$, corresponding to a wavelength of 31 cell lengths, which compares well with the actual wavelength of 33 seen in the numerical simulation with the same parameters, shown in Fig. 3. For $\kappa$ between about 0.09 and 0.345 the real part of one of the eigenvalues is positive, indicating that the homogeneous steady state is unstable to perturbations with those wavenumbers. The dispersion relation is calculated by finding the real part of the eigenvalues of the linearized system, for $\kappa$ varying upwards from zero in increments of 0.005 . 
mode in the dispersion relation is approximately $\kappa=0.2$, giving a wavelength of 31 cell lengths, in good agreement with the above observation from numerical simulations.

The critical value of the chemical diffusion coefficient at which the instability appears, denoted $D_{f}^{\text {crit }}$, depends on other parameters, in particular the influx response parameter $\sigma$, which as it changes will alter the type of steady state from which patterns may bifurcate. This dependence of $D_{f}^{\text {crit }}$ on $\sigma$ is illustrated in Fig. 6. For small $\sigma$ no spatial instability appears to be possible, intuitively because the proportion of macrophages in the tumour becomes very small, and so does any additional influx stimulated by diffusion of chemical regulators, making the response to perturbations too small to allow them to grow. For $\sigma$ large enough, patterns are always possible, with the value of $D_{f}^{\text {crit }}$ dependent on the nature of the homogeneous tumour steady state.

A further point of interest is that the wave front may be substantially altered if a spatial instability grows very quickly, so that as the normal tissue steady state is perturbed by the growing tumour, the pattern

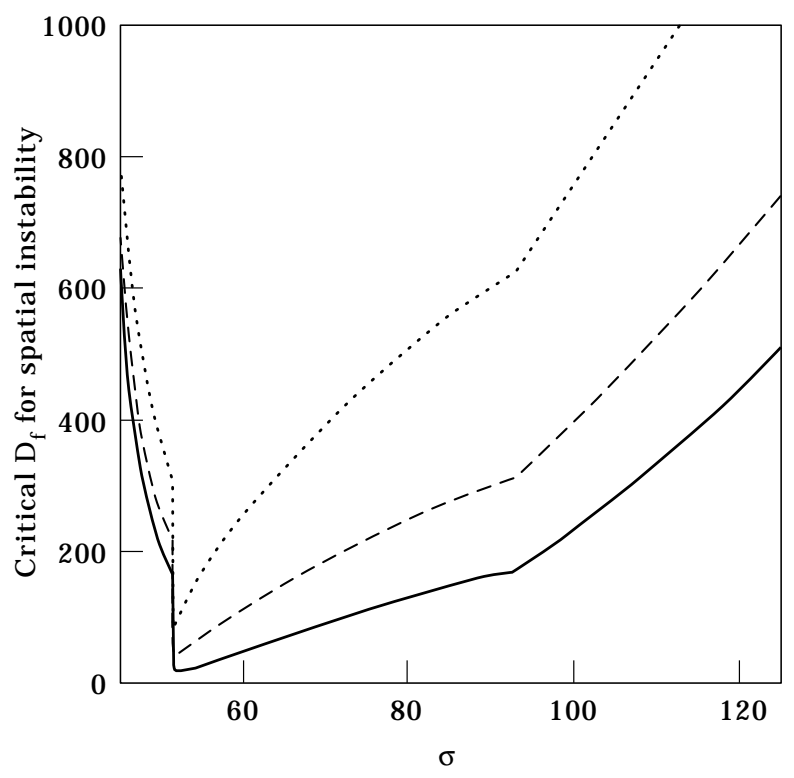

FIG. 6. The relationship between the influx response parameter $\sigma$ and the calculated critical value $D_{f}=D_{f}^{\text {crit }}$ at which the spatial instability appears. Both the full-tumour and coexistence steady states can become unstable to spatial perturbations, except that below a certain value of $\sigma$ the full-tumour steady state with a high mutant cell density is always stable, since $D_{f}^{\text {crit }}$ appears to asymptote to infinity at $\sigma \approx 36.8$. When macrophage chemotaxis is included it increases $D_{f}^{\text {crit }}$, so that increasing $\chi_{l}$ makes the whole curve move up. This means that chemotaxis can stabilize the homogeneous steady state behind the wave front. The other parameters are: $D_{l}=D_{m}=D_{n}=5, D_{c}=2.5, \alpha=0.01, \beta=5.0, \delta_{c}=0.5, \delta_{f}=2.0$, $\delta_{l}=0.1, N=1, I=0.01, k_{1}=10.0, k_{2}=0.2, \xi=2.8$.

Key: $-\chi_{1}=0 \quad--\chi_{1}=30 \quad \cdots \cdots \chi_{1}=100$ appears before a significant front can establish itself. Figure 7 shows how the wave front of the growing tumour changes as $D_{f}$ is increased far beyond $D_{f}^{\text {crit }}$. For $D_{f}<D_{f}^{\text {crit }}$ there is a simple travelling wave with a homogeneous tumour steady state behind the front. As $D_{f}$ increases, the spatial instability appears closer behind the wave front, until at very high $D_{f}$ no unpatterned region is visible.

\section{Solutions in Two Dimensions}

Thus far we have considered the model only in one space dimension, but of course real tumours reside in a three-dimensional tissue. For any mathematical model, the range of potential solution behaviours is much greater in higher dimensions, and while numerical solution of the equations in three dimensions would be too computationally intensive for our current resources, we have performed a series of two-dimensional simulations. Perhaps a central theme in the biological background to this work, and indeed in much of tumour biology, is the heterogeneity between and within tumours. The former is of particular interest at this point, since we have demonstrated a possible mechanism for the initiation of spatial heterogeneity. The more realistic two-dimensional setting, and the possibility of still greater complexity, is the focus of interest in an investigation of two-dimensional solutions.

The dispersion relation as calculated in the previous section, and illustrated in Fig. 5, is still valid, so that whenever patterns appear in one dimension they will appear in two, but the precise nature of those patterns requires investigation. In one dimension the unstable wavenumber $\kappa$ is a scalar, and a corresponding instability can only give rise to a single pattern, but in two dimensions the corresponding wavenumber $\left(\kappa_{x}, \kappa_{y}\right)$ is two-dimensional, with $\kappa=\sqrt{\kappa_{x}^{2}+\kappa_{y}^{2}}$. If one of $\kappa_{x}$ and $\kappa_{y}$ is zero then the pattern will be just simple stripes, but if they are both non-zero then the pattern will be two-dimensional, tiling the plane. This introduces the possibility of more than one pattern being stable for a given parameter set, depending on the domain and initial conditions.

Figure 8 shows a shaded plot of the macrophage density for a simulation starting with initial conditions of a seeding region of mutant cells at the centre, with the rest of the domain at the normal tissue steady state. A circular travelling wave is established, behind which the tumour steady state is unstable to spatial perturbations, which leads to the formation of a stationary target pattern. The macrophage density is shown at dimensionless times $40,60,80$, and 100 to illustrate the evolution of the 

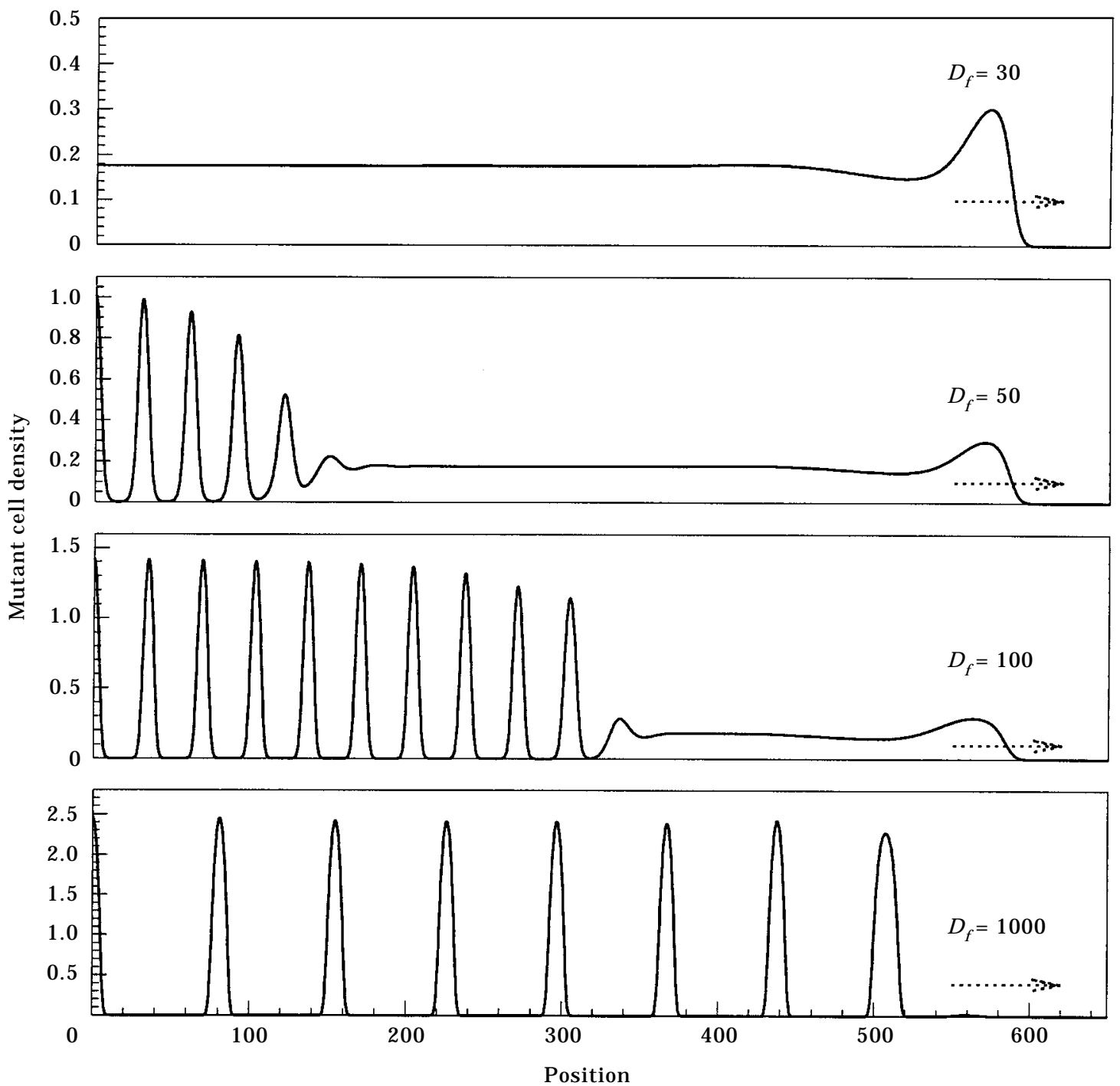

FIG. 7. Numerical solutions of the model (A.1) at dimensionless time 100 for varying $D_{f}$. From top to bottom, $D_{f}=30,50,100,1000$. As $D_{f}$ increases through a critical value the tail of the wave becomes unstable to spatial perturbations, which form some distance behind the tail of the wave front for $D_{f}$ not too big, but as it increases further the pattern appears as soon as the front perturbs the solution away from the normal tissue state. The wave front itself becomes almost non-existent, so that it becomes important to consider the implications of very low densities in the context of a differential equation model. The other parameters are as in Fig. 5.

wave and pattern. The concentric rings in the circular geometry of the simulation correspond to stripes in a planar geometry, and simulations for the same parameters do indeed give simple stripes behind a planar wave front (omitted for brevity). In real tumours other factors mean that any heterogeneity would be much more irregular. Figure 9 shows the macrophage density for a simulation where the initial conditions are a random perturbation of the normal tissue steady state on the whole of a square domain. Because there is no prepattern laid down by the travelling wave, the solution is not forced to a particular orientation, and so even after very long times, the solution has not settled down, although some semblance of a striped pattern is clear.

Figure 10 shows the macrophage density for a simulation for different values of $\sigma$ and $D_{f}$, where the pattern that develops behind the wave front is one of spots aligned like the squares on a chessboard. Close to the pattern forming bifurcation either spots or stripes - but not both - are stable (Ermentrout, 1991), but it is not possible to exclude the possibility of more than one stable pattern when the parameters are not close to the bifurcation. Indeed, further simulations not illustrated here show that different patterns such as spots and stripes can stably coexist for certain parameter values, with 

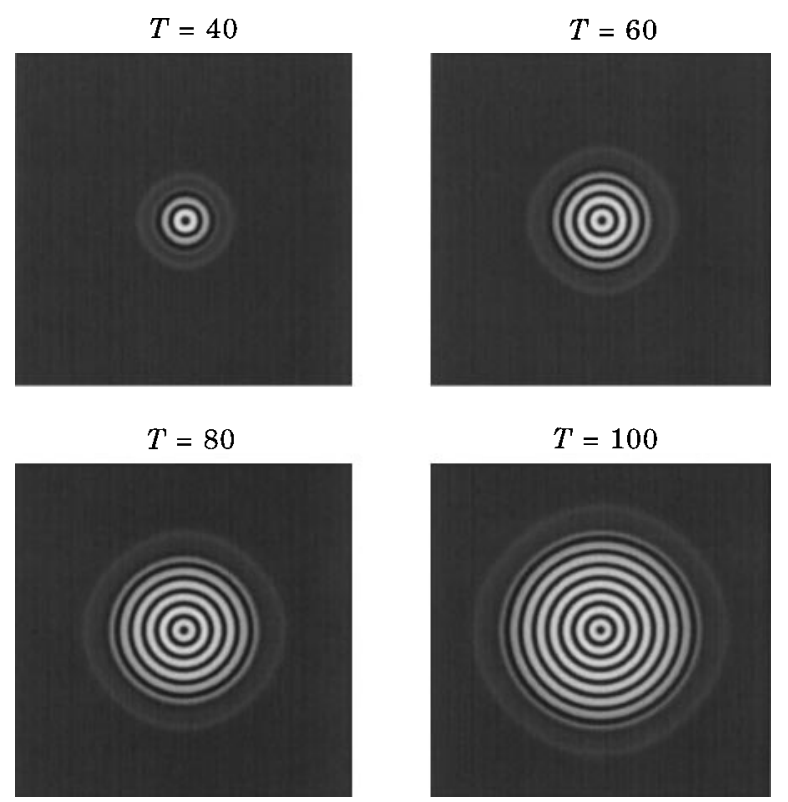

FIG. 8. Numerical simulation in two dimensions, showing the evolution of the macrophage density at intervals of 20 dimensionless time units. Initial conditions were a unit seeding region with a mutant cell density of 1 , located at the centre of a domain at the normal tissue steady state. A target patten appears behind the wave-front of invading mutant cells. The domain size is $1200 \times 1200$, and the parameters are: $D_{l}=D_{m}=D_{n}=5$, $D_{f}=250, D_{c}=2.5, \alpha=0.01, \beta=5.0, \delta_{c}=0.5, \delta_{f}=2.0, \delta_{l}=0.1$, $N=1, I=0.01, k_{1}=10.0, k_{2}=0.2, \sigma=30, \xi=2.0$. The model equations were solved in two dimensions using an alternating direction implicit method. The boundary conditions used were zero-flux in all simulations.

the initial conditions determining which state is reached.

While one does not expect to find such regular patterns in real tumours, we have shown that the macrophage-tumour interaction could initiate heterogeneity, and of course a wide variety of other factors would affect the process so that the end result could be very irregular. Indeed, there is scope for further work which could incorporate the effects of noise and parameter variations, physical differences in the tissue structure, and other cellular interactions.

The shape of the tumour boundary is an important indicator of the capability of a tumour to invade the tissue surrounding it. Clinical diagnosis has relied upon a subjective assessment of the complexity of the boundary, but the application of techniques from fractal geometry has begun to provide a more formal basis for diagnosis (Landini \& Rippin, 1996). The projection of "fingers" of cells from the tumour mass into the surrounding tissue indicates an invasive tumour, and mathematical models for tumour growth have been used to investigate possible mechanisms for tumour fingering (Byrne \&

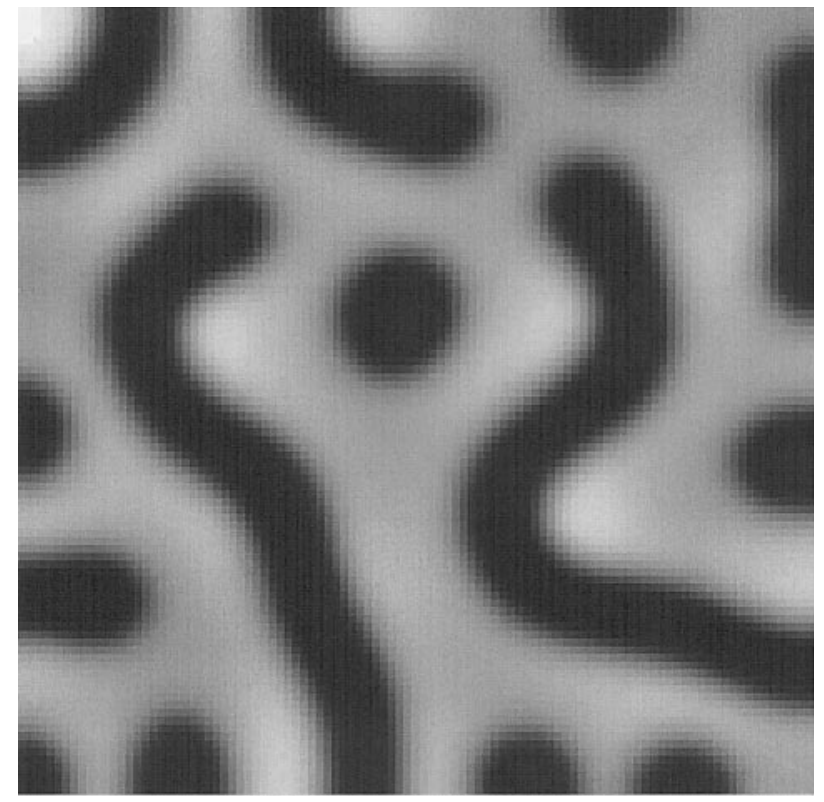

FIG. 9. Numerical simulation in two dimensions, showing the macrophage density after 1000 dimensionless time units of evolution from initial conditions with a random perturbation of the normal tissue steady state throughout a square domain. The domain size is $200 \times 200$, and the parameters are as in Fig. 8 .

Chaplain, 1996; Chaplain, 1993). Simulations of our model suggest that the tumour-macrophage interaction, with random cell motion, is not a mechanism for fingering.

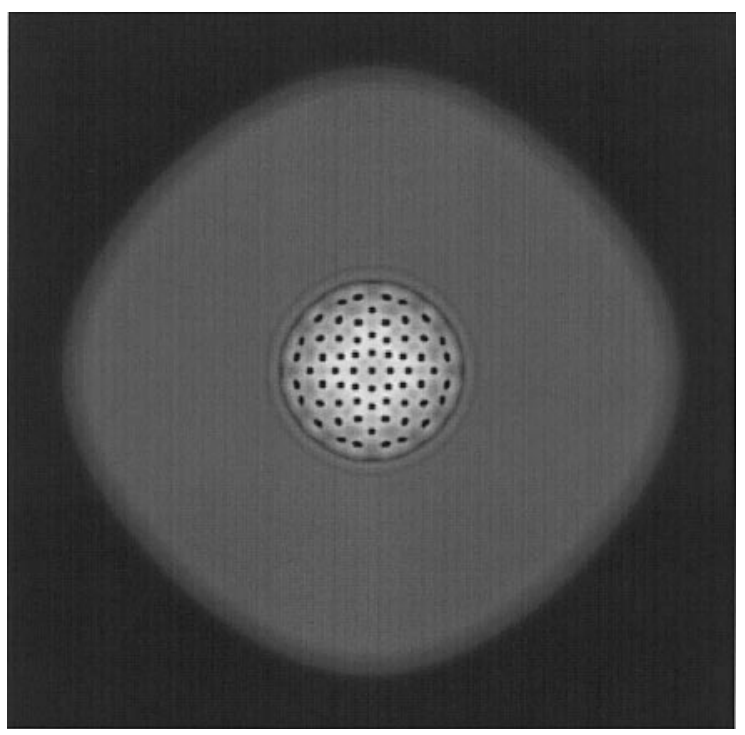

FIG. 10. Numerical simulation in two dimensions of the model (A.1), showing the macrophage density after 200 dimensionless time units of evolution from a unit seeding region with a mutant cell density of 1 , located at the centre of the domain. Behind the wavefront a spatial perturbation grows to form a chessboard type tiling of the plane. The domain size is $2400 \times 2400$ dimensionless units, and the parameters are as for Fig. 8 except for $D_{f}=750$ and $\sigma=58$. 


\section{Macrophage Chemotaxis: Estimation of Motility Parameters}

In the preceding sections we have discussed only the implications of random cell movement for spatial tumour growth in the presence of macrophages. In this section we describe the estimation of the parameters governing macrophage chemotaxis, using experimental data, before proceeding to describe the types of solutions seen when chemotaxis is included in the model equations. The terms for macrophage movement in the full model are of Keller-Segel form (Keller \& Segel, 1971a,b). It is well known that chemoattractants often stimulate random as well as directed cell movement, in a concentration - as well as gradient-dependent fashion, so that it is to be expected that the diffusion and chemotaxis parameters will vary with chemical concentration. A particularly common type of in vitro system used to test such effects is the Boyden chamber (Bignold, 1988; Boyden, 1962), consisting of two wells separated by a filter. The cells under test are induced to move through this filter by filling the upper and lower wells with varying concentrations of chemoattractant, thereby setting up a gradient. Random cell motion can be isolated by making the concentrations in both wells the same, so that there is no chemical gradient across the filter. After a suitable period, the filter is removed and the number of cells that have migrated across the filter is counted. It is important to note that there may be significant differences between such in vitro experiments and the in vivo situation, since a polycarbonate filter is a very different material from in vivo extracellular matrices. The key property of macrophage motility is the relative contributions of random and directed movement, and we assume that this property will be approximately conserved in a variety of different environments.

Following work by Sherratt et al. (1993), the Boyden chamber assay is modelled by two equations, for the migration of macrophages $l(x, t)$ and the diffusion of the chemical $f(x, t)$ :

$$
\begin{gathered}
\frac{\partial l}{\partial t}=\frac{\partial}{\partial x}\left[D_{l}(f) \frac{\partial l}{\partial x}\right]-\frac{\partial}{\partial x}\left[\chi_{l}(f) l \frac{\partial f}{\partial x}\right] \\
\frac{\partial f}{\partial t}=D_{f} \frac{\partial^{2} f}{\partial x^{2}} .
\end{gathered}
$$

Note that macrophage movement is again formulated with the Keller-Segel approach. Cell migration is limited to the filter, which has width $a$, but the chemical diffuses throughout the upper and lower wells, giving a much larger domain, which can be considered infinite to a good approximation. Note that we neglect cell division because the duration of the experiments is much less than typical cell cycle times. The other parameters for the system are the filter area $A$, and the area $A_{\text {count }}$ in which migrated cells are counted; macrophage length $L$; initial number of cells added $M_{0}$, and initial cell density $l_{0}$; initial concentration in the lower well $f_{L}$, and upper well $f_{U}$; and final time $T$. Assuming the cells form a monolayer of thickness $L$ on the upper surface of the filter, $l_{0}=M_{0} / A L$. The initial conditions are therefore $l=0$ on $0 \leqslant x<a$ and $l=l_{0}$ at $x=a$. Cells adhere to the surface of the filter and do not drop off, so that the surface densities change at a rate corresponding to the cell flux. Thus, the following boundary conditions hold:

$$
\begin{aligned}
\left.\frac{\partial l}{\partial t}\right|_{x=0} & =\frac{D_{l}}{L} \frac{\partial l}{\partial x}-\frac{\chi_{l}}{L} l \frac{\partial f}{\partial x}, \\
\left.\frac{\partial l}{\partial t}\right|_{x=a} & =-\frac{D_{l}}{L} \frac{\partial l}{\partial x}+\frac{\chi_{l}}{L} l \frac{\partial f}{\partial x}, \\
f(-\infty, 0) & =f_{L}, \quad f(+\infty, 0)=f_{U} .
\end{aligned}
$$

The diffusion equation for $f$ is straightforward to solve analytically. Note that the concentration at the bottom of the filter is constant: $f(0)=\left(f_{U}+f_{L}\right) / 2$. Since $f$ varies by less than $1 \%$ across the filter within 2 minutes of the start of the experiment, we take $D_{l}$ and $\chi_{l}$ to be constant for a given experiment. Similarly, the change in gradient across the filter is negligible, giving the approximate expression:

$$
\frac{\partial f}{\partial x} \approx \frac{f_{U}-f_{L}}{2 \sqrt{\pi D_{f} t}} .
$$

Numerical simulations of the model (6) were carried out, using this approximation to the chemical gradient, and a comparison of model predictions and experimental data was used to determine the motility parameters. A common pattern of experimental data which is ideally suited to such a determination is a checkerboard analysis, where a range of chemical concentrations is tested, using every possible combination in the upper and lower wells. This will then include cases where the upper and lower concentrations are equal, so that the random motility coefficient may be isolated. The data considered here is from Sozzani et al. (1991), which is reproduced in Table 1. Note that the data is for the migration of monocytes obtained from blood donors. Monocytes are the precursors in blood of macrophages, and we are not aware of any data on the differences there may 
TABLE 1

Calculated mobility parameters for macrophages responding to $M C P-1$

\begin{tabular}{ccccccc}
\hline $\begin{array}{l}\text { MCP-1 }\left(\times 10^{-10} \mathrm{M}\right) \\
\text { Lower Well }\end{array}$ & Upper Well & Filter & $\begin{array}{c}\text { Cells } \\
\text { counted }\end{array}$ & $\begin{array}{c}D_{l} \\
\left(\mathrm{~m}^{2} \mathrm{~s}^{-1}\right)\end{array}$ & $\begin{array}{c}\chi_{l} \\
\left(\mathrm{~m}^{2} \mathrm{~s}^{-1} \mathrm{M}^{-1}\right)\end{array}$ & $\begin{array}{c}\chi_{l} \times \text { filter } \\
\operatorname{conc}^{n}\left(\mathrm{~m}^{2} \mathrm{~s}^{-1}\right)\end{array}$ \\
\hline 0 & 0 & 0 & $20 \pm 3$ & $4.61 \times 10^{-15}$ & 0 & 0 \\
1 & 1 & 1 & $57 \pm 8$ & $1.04 \times 10^{-14}$ & 0 & 0 \\
5 & 5 & 5 & $36 \pm 6$ & $6.82 \times 10^{-15}$ & 0 & 0 \\
10 & 10 & 10 & $12 \pm 1$ & $3.54 \times 10^{-15}$ & 0 & 0 \\
1 & 0 & 0.5 & $48 \pm 3$ & $7.51 \times 10^{-15}$ & 0.00622 & $3.11 \times 10^{-13}$ \\
5 & 0 & 2.5 & $62 \pm 7$ & $9.06 \times 10^{-15}$ & 0.00198 & $4.95 \times 10^{-13}$ \\
10 & 0 & 5 & $75 \pm 4$ & $6.82 \times 10^{-15}$ & 0.00259 & $12.95 \times 10^{-13}$ \\
\hline
\end{tabular}

$D_{l}$ was first estimated for the cases with equal concentrations in the upper and lower wells by finding the value for which numerical simulations predicted the correct number of cells counted. $D_{l}$ can be isolated in this way because in these situations there will be no chemical gradient and hence no chemotaxis. Using these calculated values, $\chi_{l}$ can be estimated in the same way. The experimental data is taken from Boyden Chamber experiments of Sozzani et al. (1991), showing the number of migrated monocytes counted in five oil fields, for different concentrations of recombinatant MCP-1 in the upper and lower wells. $50 \mu \mathrm{l}$ of cell suspension $\left(1.5 \times 10\right.$ monocytes $\left.\mathrm{ml}^{-1}\right)$ was seeded in the upper compartment. The polycarbonate filter had a pore size of $5 \mu \mathrm{m}$ and we assumed the standard width of $10 \mu \mathrm{m}$, and area of $7 \times 10^{-6} \mathrm{~m}^{2}$. The chambers were incubated at $37^{\circ} \mathrm{C}$ in air with $5 \% \mathrm{CO}_{2}$ for $90 \mathrm{~min}$. Filters were then removed, fixed, and stained with Diff-Quik, and five high power oil-immersion fields were counted. Note that the molecular weight of MCP-1 means that $1 \mathrm{ng} \mathrm{ml}{ }^{-1} \approx 10^{-10} \mathrm{M}$.

be between monocyte and macrophage migration. We assume that although the actual values of their motility parameters may change, the relative contributions of random and directed movement will be similar.

The experiments used the monocyte chemoattractant MCP-1, discussed in Section 1-its diffusion coefficient in aqueous solution is estimated using standard Stokes-Einstein theory, using

$$
D_{f}=\frac{k T}{6 \pi \eta} \cdot\left[\frac{4 \pi N_{A}}{3 M v}\right]^{1 / 3}
$$

Here $k$ is the Boltzmann constant; $T$ is absolute temperature, and $\eta$ is the viscosity of water that temperature; $v$ is the specific volume and $M$ is the molecular weight of the solute; and $N_{A}$ is Avogadro's number. There are a number of different values quoted in the literature for the molecular mass of MCP-1, ranging from 10 to $16 \mathrm{kD}$ (Bottazzi et al., 1985; Graves et al., 1989; Mantovani, 1990; van Damme et al., 1992; Walter et al., 1991). Bearing this in mind, the formula gives an approximate value for $D_{f}$ of $2 \times 10^{-10} \mathrm{~m}^{2} \mathrm{~s}^{-1}$.

Estimates for the monocyte motility parameters were made by varying the model parameters to match the predicted monocyte density at the bottom of the filter with the actual number of cells counted in experiments. $D_{l}$ was first estimated for the cases with equal concentrations in the upper and lower wells by finding the value of $D_{l}$ for which numerical simulations predicted the correct number of cells counted. $D_{l}$ can be isolated in this way because in these situations there will be no chemical gradient and hence no chemotaxis. Using these calculated values, $\chi_{l}$ can be estimated in a similar fashion. The results are presented in Table 1, and Fig. 11 shows an example of a numerical simulation of monocyte migration across the filter.

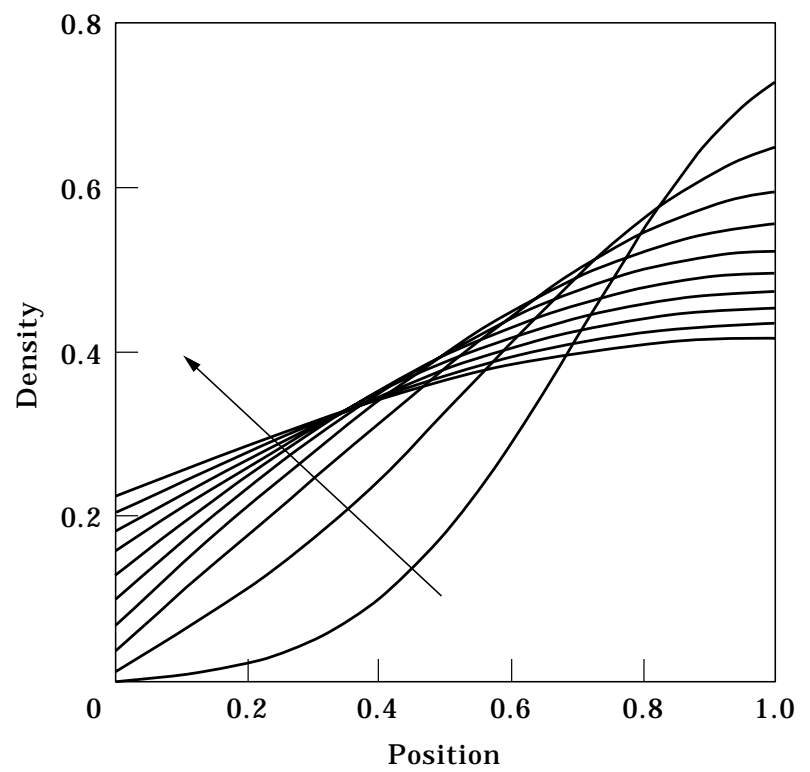

FIG. 11. Numerical simulation of the migration of monocytes across a polycarbonate filter in response to a chemical gradient of the chemoattractant MCP-1, using the model (6). Estimates for the monocyte motility parameters were made by varying the model parameters to match the model's predicted monocyte density at the bottom of the filter $(x=0)$ with the actual number of cells counted in experiments. The solution is plotted at intervals of 9 minutes, up to a final time of 90 minutes. The parameters were $D_{l}=6.82 \times 10^{-15}$ and $\chi_{l}=0.00259$, which gives a predicted number of migrated cells of 75 , compared with the experimental count of $75 \pm 4$ cells. 
Using the calculated values from Table 1, we fix ranges for the dimensionless values of $D_{l}$ and $\chi_{l}$. The random motility coefficient varies from $4.61 \times 10^{-15}$ to $1.04 \times 10^{-14} \mathrm{~m}^{2} \mathrm{~s}^{-1}$, and applying the non-dimensionalization in the Appendix gives $D_{l} \in[23,52]$. Similarly the calculated range for the chemotaxis coefficient was from 0.00198 to $0.00622 \mathrm{~m}^{2} \mathrm{~s}^{-1} \mathrm{M}^{-1}$, giving $\chi_{l} \in[990,3110]$. Kuratsu et al. (1993) measured MCP-1 levels in a number of patients both with and without tumours, finding a range of concentrations in vivo of $0.2-6.3 \mathrm{ng} \mathrm{m}^{-1}$, which compares well with the range for the in vitro experimental data of $0.5-10 \mathrm{ng} \mathrm{ml}^{-1}$. Thus, the dimensionless motility parameters we have calculated are appropriate for our model of in vivo tumour growth.

\section{Simulations with Macrophage Chemotaxis}

An important feature of the inclusion of chemotaxis in the model is the possibility that it can stabilise

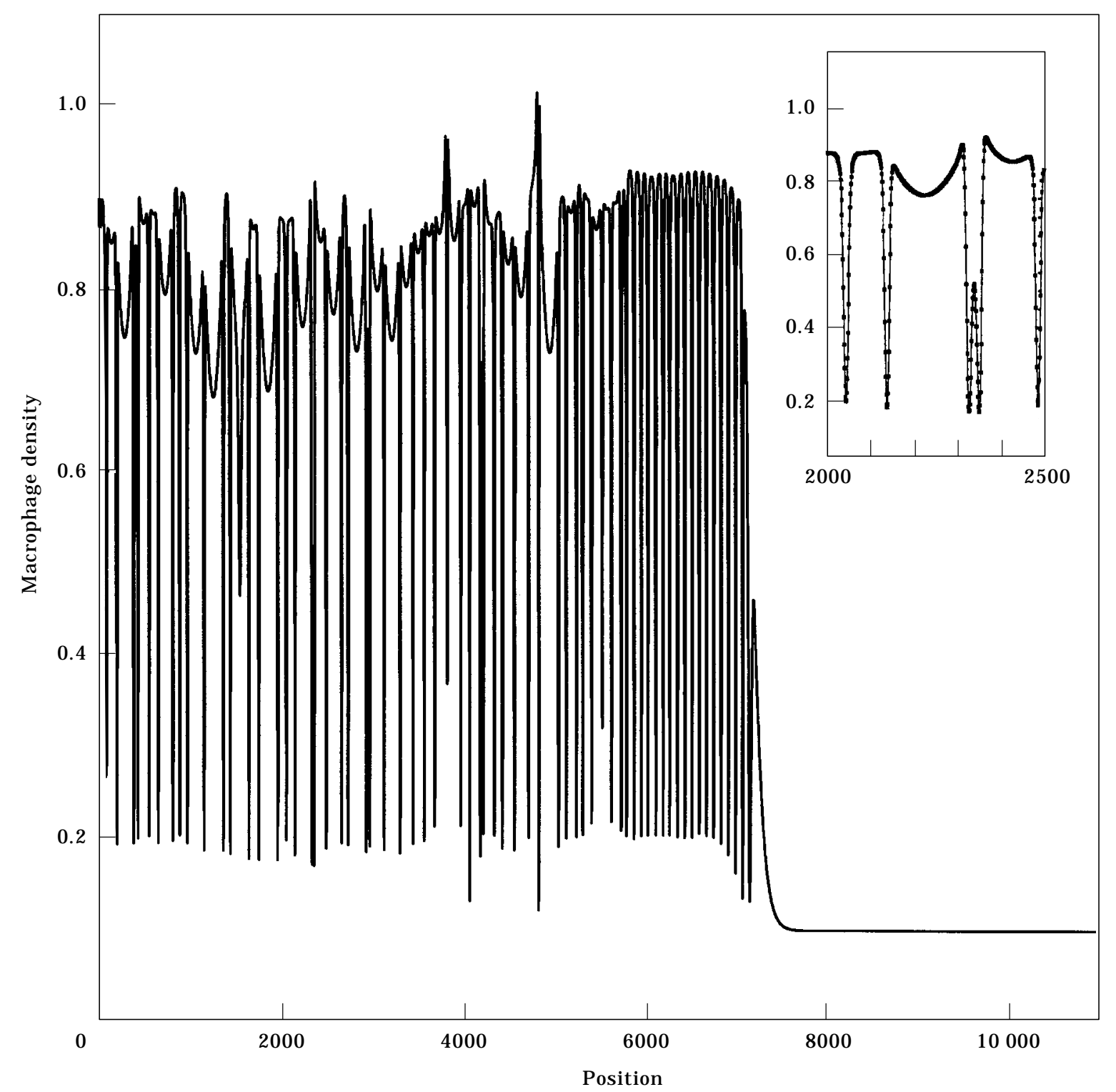

FIG. 12. Numerical simulation of the model (A.1) using motility parameters estimated from the Boyden chamber data of Sozzani et al. (1991). The solution in space is shown after 1200 dimensionless time units of evolution from initial conditions corresponding to a mutation at the left hand boundary of the domain. Macrophage chemotaxis causes the previously regular pattern behind the wave front to become irregular in both space and time (not illustrated here). The magnification shows the individual grid points of the finite difference numerical scheme, illustrating the spatial resolution of the solution. The parameters are: $D_{l}=40, D_{m}=D_{n}=5, D_{f}=10000, D_{c}=2.5, \chi_{l}=2000$, $\alpha=0.01, \beta=5.0, \delta_{c}=0.5, \delta_{f}=2.0, \delta_{l}=0.1, N=1, I=0.01, k_{1}=10.0, k_{2}=0.2, \xi=2.8, \sigma=58$. 
the spatially homogeneous steady state when this is unstable to perturbations in the absence of chemotaxis. Examination of the dispersion relation shows that as the chemotaxis coefficient is increased the peak in the dispersion relation moves down, so that wavenumbers whose corresponding eigenvalues had a positive real part no longer do so. This means that as $\chi_{l}$ increases, the value of the chemical diffusion coefficient at which spatial instabilities appear, denoted $D_{f}^{\text {crit }}$, also increases. This is demonstrated in Fig. 6 which shows $D_{f}^{\text {crit }}$ as $\sigma$ varies, for $\chi_{l}$ increasing from zero. As the chemotaxis coefficient is increased, the whole curve of critical $D_{f}$ is moved up, demonstrating the effect over a range of parameters which includes both types of tumour steady state.

In contrast, some simulations with chemotaxis show more complicated dynamics behind the wave front, with irregular spatiotemporal variations. Figure 12 shows the result of such a simulation, for macrophage motility parameters within the ranges calculated in the previous section. Behind the wave front the solution does not settle down either in time or in space.

A mathematical discussion of the mechanism responsible for these irregular solutions will be presented elsewhere, but we will summarise a few points here. The oscillations are not due to any oscillatory behaviour in the kinetics, in contrast to work by others such as Dunbar (1986), Sherratt et al. (1995), and Merkin et al. (1996), which is concerned with reaction-diffusion systems whose kinetics are oscillatory in the absence of spatial variations. Using the domain length as a parameter, we found that it is the pattern itself which becomes unstable through a bifurcation to a new oscillating solution branch. We hypothesised that this bifurcation would repeat at integer multiples of the critical domain length, and numerical solutions provided evidence for this. This explains the irregular wakes seen behind travelling wave fronts in simulations on large domains-as the wave progresses, oscillatory solutions continually appear and disappear as the effective "domain length" moves through a series of bifurcation points. Note that the long-term behaviour of the solutions on a fixed finite domain is not irregular, but oscillatory with a constant period.

\section{Discussion}

Travelling waves in models of tumour invasion have been demonstrated previously, but there has been little investigation of spatial instabilities within growing tumours. We have shown that the rapid diffusion of chemical regulators in comparison to cell movement, combined with the anti-tumour effects of macrophages, can lead to the onset of instability and the appearance of hot-spots of tumour cell density. In real tumours a variety of other factors mean that the regular patterns seen in one and two dimensional simulations do not occur. However, there is evidence for the existence of dense clusters of macrophages, whose location is thought to be related to the tumour-cytotoxic role of macrophages (Kerrebijn et al., 1994). This supports our view that the interaction between macrophages and tumour cells is a potential mechanism for generating spatial heterogeneity. In the 1950s and 1960s, the potential of the immune system to spontaneously eliminate tumours was widely debated - e.g. Baldwin (1955); more recently, this "immune surveillance hypothesis" has been widely disputed and remains controversial. In recent years, attention has switched to more complex regulatory effects of the immune system on tumour progression (Matzinger, 1994), including the tumourpromoting role of immune system cells-for example, recent evidence that macrophages promote angiogenesis in breast carcinomas (Lewis et al., 1995). Our work provides a link between these old and new philosophies. We have shown that while the tumouricidal activity of macrophages is insufficient to eliminate tumours, it can have important implications for their structure.

Note that, in contrast to some other models for biological pattern formation, there is no prepattern involved, and no mechanical interactions are included. We expect that the simple combination of chemical diffusion and the much slower movement of cells will generate similar phenomena in a variety of biological situations.

It is important to consider the role of very low population densities within the context of a partial differential equation model. When high chemical diffusion causes the wave front to be extremely small, as illustrated in Fig. 7, it could be argued that such low densities should be considered to be zero. Other models have used a threshold below which densities are taken to be zero (Mollison \& Daniels, 1993), which would have considerable implications on the biological interpretation of our model. For example, if such a threshold were applied then for sufficiently high chemical diffusion coefficients only the first peak of a pattern would appear, and then the wave of invading mutant cells would be halted. Intuitively, if the chemical regulator did diffuse fast enough, it would always activate macrophages outside the tumour boundary, preventing further outgrowth. This represents a situation where tumour growth is 
held in check by the immune system, although the tumour itself is not eliminated.

In Section 7 we described the estimation of the parameters governing macrophage motility using Boyden chamber data from a paper by Sozzani et al. (1991). Data from different in vitro systems will yield different values for motility parameters, but the key issue is not the precise numbers but the relative contributions of random and directed cell migration. Although it can be argued in this way that different assays will preserve the ratios of certain coefficients, there may be further differences between the in vivo and in vitro situations that are dependent not on the experimental system but on the cells being tested themselves. Phenotypic changes may be induced by the transition to an in vitro environment, so that the behaviour of cells can be markedly different from in vivo. A simple example of such an effect is found in a different context, the fibroblast-populated collagen lattice (Grinnell \& Lamke, 1984), which is an in vitro representation of wound contraction. Here, dermal fibroblasts change phenotype, when placed in an in vitro collagen gel, to resemble the contractile "myofibroblast" found in real wounds.

In the final section we demonstrated that chemotaxis can stabilise the homogeneous tumour steady state behind the wave, but can also initiate spatiotemporally irregular solutions. These results raise a key question about the effect of chemotaxis in the more realistic two and three dimensional settings. What kind of irregular patterns may we expect to see? Can chemotaxis affect the stability of the wavefront? We believe this work is important because this is the first explicit model mechanism which leads naturally to irregularity in tumour composition.

Do solutions showing these spatiotemporal irregularities have any biological relevance? There is ample evidence of spatial heterogeneity in tumours, but detailed spatiotemporal data is unavailable. Recent biological developments indicate that the proportion of macrophages in a tumour is closely correlated with prognosis, and that macrophages may be linked to a complex spatiotemporal variation in blood supply and tissue oxygenation. Macrophage hot spots are situated away from regions of high vascular density, implying that macrophages actively migrate away from the blood vessels (Leek et al., 1996). Macrophages themselves promote angiogenesis (Lewis et al., 1995), so that they will encourage new vascular hot spots to develop. If the macrophages then move away or die, then we have the ingredients for a constantly changing spatiotemporal picture. Although the model described in this paper does not include interactions with the tumour vasculature, future research will depend upon an understanding of the patterning and irregular behaviour that we have described.

M.R.O. was supported by an earmarked graduate studentship from the Engineering and Physical Sciences Research Council of Great Britain. This work was supported in part by a grant from the London Mathematical Society. We thank Claire Lewis, Abbey Perumpanani, Nigel Burroughs and Mark Lewis for helpful discussions. Part of this work was done during visits by M.R.O. and J.A.S. to the University of Utah, as part of the Special Year in Mathematical Biology.

\section{REFERENCES}

Adam, J. A. \& Bellomo, N. (1997). A Survey of Models for Tumor-immune System Dynamics. Berlin: Birkhäuser.

AdAM, J. A. (1993). The dynamics of growth-factor-modified immune response to cancer growth: One dimensional models. Mathl. Comp. Modelling 17, 83-106.

Adams, D. O. \& Hamilton, T. A. (1984). The cell biology of macrophage activation. Ann. Rev. Immunol. 2, 283-318.

Albert, A., Freedman, M. \& Perelson, A. S. (1980). Tumors and the immune system: The effects of a tumor growth modulator. Math. Biosci. 50, 25-28.

BALDWIN, R. W. (1955). Immunity to methylcholanthrene induced tumours in inbred rats following atrophy and regression of implanted tumours. Br. J. Cancer 9, 652-657.

BignOLD, L. P. (1988). Measurement of chemotaxis of polymorphonuclear leukocytes in vitro. J. Immunol. Meth. 108, 1-18.

Bottazzi, B., Polentarutti, N., Acero, R., Balsari, A., BoRASCHI, D., GHezzi, P. et al. (1983). Regulation of the macrophage content of neoplasms by chemoattractants. Science 220, 210-212.

Bottazzi, B., Ghezzi, P., Taraboletti, G., Salmona, M., Colombo, N., Bonnazzi, C. ET AL. (1985). Tumor-derived chemotactic factor(s) from human ovarian carcinoma: Evidence for a role in the regulation of macrophage content of neoplastic tissues. Int. J. Cancer. 36, 167-173.

Bottazzi, B., Erba, E., Nobili, N., Fazioli, F., Rambaldi, A. \& Mantovani, A. (1990). A paracrine circuit in the regulation of the proliferation of macrophages infiltrating murine sarcomas. $J$. Immunol. 144, 2409-2412.

Bottazzi, B., Walter, S., Govoni, D., Colotta, F. \& Mantovani, A. (1992). Monocyte chemotactic cytokine gene transfer modulates macrophage infiltration, growth, and susceptibility to IL-2 therapy of a murine melanoma. J. Immunol. 148, $1280-1285$

Boyden, S. V. (1962). The chemotactic effect of mixtures of antibody and antigen on polymorphonuclear leukocytes. $J$. Exp. Med. 115, 453-466.

Byrne, H. M. \& Chaplain, M. A. J. (1996). Modelling the role of cell-cell adhesion in the growth and development of carcinoma. Mathl. Comp. Modelling 24, 1-17.

Chaplain, M. A. J. (1993). The development of a spatial pattern in a model for cancer growth. In: Experimental and Theoretical Advances in Biological Pattern Formation (Othmer, H. G., Maini, P. K. \& Murray, J. D., eds), pp. 45-60. New York: Plenum Press. Chaplain, M. A. J. \& Sleeman, B. D. (1993). Modelling the growth of solid tumours and incorporating a method for their classification using nonlinear elasticity theory. J. Math. Biol. 31, 431-479.

Dunbar, S. R. (1986). Traveling waves in diffusive predator-prey equations - periodic orbits and point-to-periodic heteroclinic orbits. SIAM J. Appl. Math. 46, 1057-1078.

ERmentrout, B. (1991). Stripes or spots-nonlinear effects in bifurcation of reaction-diffusion equations on the square. Proc. R. Soc. Lond. A 434, 413-417. 
Esgro, J. J., Whitworth, P. \& Fidler, I. J. (1990). Macrophages as effectors of tumor immunity. Immunol. Allergy Clin. N. Am. 10, 705-729.

Fearon, E. R. \& Vogelstein, B. (1990). A genetic model for colorectal tumorigenesis. Cell 61, 759-767.

Graves, D. T., Jiang, Y. L., Williamson, M. J. \& Valente, A. J. (1989). Identification of monocyte chemotactic activity produced by malignant cells. Science 245, 1490-1493.

GrEensPan, H. P. (1972). Models for the growth of a solid tumour by diffusion. Stud. Appl. Math. 51, 317-340.

GrinNell, F. \& LAMKE, C. R. (1984). Reorganisation of hydrated collagen lattices by human fibroblasts. J. Cell. Sci. 66, 51-63.

Hamilton, T. A. \& Adams, D. O. (1987). Mechanisms of macrophage-mediated tumor injury. In: Tumor ImmunologyMechanisms, Diagnosis, Therapy (den Otter, W. \& Ruitenberg, E. J., eds), pp. 89-107. Oxford: Elsevier Science Publishers.

HibBs, J. B. (1991). Synthesis of nitric-oxide from 1-arginine-a recently discovered pathway induced by cytokines with antitumor and antimicrobial activity. Res. Immunol. 142, $565-569$.

Jonjic, N., Alberti, S., Bernasconi, S., Peri, G., Jilek, P., Anichini, A., Parmiani, G. \& Mantovani, A. (1992). Heterogeneous susceptibility of human-melanoma clones to monocyte cytotoxicity - role of ICAM-1 defined by antibody blocking and gene transfer. Eur. J. Immunol. 22, 2255-2260.

Keller, E. F. \& Segel, L. A. (1971a). Model for chemotaxis. J. theor. Biol. 30, 225-234.

Keller, E. F. \& Segel L. A. (1971b). Travelling bands of chemotactic bacteria: A theoretical analysis. J. theor. Biol. 30, 235-248.

KeLLER, R. (1993). The macrophage response to infectious agents: Mechanisms of macrophage activation and tumour cell killing. Res. Immunol. 144, 271-273.

Kerrebijn, J. D., Balm, A. J. M., Knegt, P. P., Meeuwis, C. A. \& Drexhage, H. A. (1994). Macrophage and dendritic cell infiltration in head and neck squamous-cell carcinoma-an immunohistochemical study. Cancer Immunol. Immunotherapy 38, 31-37.

Kuratsu, J., Yoshizato, K., Yoshimura, T., Leonard, E. J., Takeshima, H. \& Ushio, Y. (1993). Quantitative study of monocyte chemoattractant Protein-1 (MCP-1) in cerebrospinal fluid and cyst fluid from patients with malignant glioma. J. Nat. Cancer Inst. 85, 1836-1839.

Kuznetsov, V. A., Zhivoglyadov, V. P. \& Stepanova, L. A. (1993). Kinetic approach and estimation of the parameters of cellular interaction between the immunity system and a tumor. Arch. Immunol. Ther. Exp. 41, 21-31.

Kuznetsov, V. A., Makalkin, I., Taylor, M. A. \& Perelson, A. S. (1994). Nonlinear dynamics of immunogenic tumors: Parameter estimation and global bifurcations analysis. Bull. Math. Biol. 56, 295-321.

LANDinI, G. \& Rippin, J. W. (1996). How important is tumor shape - quantification of the epithelial connective-tissue interface in oral lesions using local connected fractal dimension analysis. J. Path. 179, 210-217.

Leek, R. D., Lewis, C. E., Whitehouse, R., Greenall, M., Clarke, J. \& HARRIS, A. L. (1996). Association of macrophage infiltration with angiogenesis and prognosis in invasive breast carcinoma. Cancer Res. 56, 4625-4629.

Lefever, R., Hiernaux, J., Urbain, J. \& Meyers, P. (1992). On the kinetics and optimal specificity of cytotoxic reactions mediated by T lymphocyte clones. Bull. Math. Biol. 54, 839-873.

Lewis, C. E., Leek, R., Harris, A. \& McGee, J. O. D. (1995). Cytokine regulation of angiogenesis in breast cancer - the role of tumor-associated macrophages. J. Leukoc. Biol. 57, $747-751$.

Mace, K. F., Ehrke, M. J., Hori, K., Maccubbin, D. L. \& Minich, E. (1988). Role of tumor necrosis factor in macrophage activation and tumoricidal activity. Cancer Res. 48, 5427-5432.

Mantovani, A. (1989). Cytotoxic killing of tumour cells by monocytes. In: Human Monocytes (Zembala, M. \& Asherson, C. L., eds), pp. 303-311. London: Academic Press.
Mantovani, A. (1990). Tumour-associated macrophages. Curr. Opin. Immunol. 2, 689-692.

Mantovani, A. (1993). Discussion: 50th forum in immunologymacrophages and cancer. Res. Immunol. 144, 296.

Mantovani, A., Bottazzi, B., Colotta, F., Sozzani, S. \& Ruco, L. (1992). The origin and function of tumor-associated macrophages. Immunol. Today 13, 265-270.

Markovitch, S. (1993). The particular role of cell loss in tumor growth. Mathl. Comp. Modelling 18, 83-89.

Matzinger, P. (1994). Tolerance, danger, and the extended family. Annu. Rev. Immunol. 12, 991-1045.

Merkin, J. H., Petrov, V., Scott, S. K. \& Showalter, K. (1996). Wave-induced chemical chaos. Phys. Rev. Lett. 76, 546-549.

Michelson, S. \& Leith, J. (1991). Autocrine and paracrine growth factors in tumor growth: A mathematical model. Bull. Math. Biol. 53, 639-656.

Mollison, D. \& Daniels, H. (1993). The deterministic simple epidemic unmasked. Math. Biosci. 117, 147-153.

O'Sullivan, C. \& Lewis, C. E. (1994). Tumor-associated leukocytes-friends or foes in breast-carcinoma. J. Path. 172, 229-235.

Owen, M. R. \& SHERRATt, J. A. (1997). Modelling the macrophage invasion of tumours: effects on growth and composition. IMA J. Math. Appl. Biol. Med., (in press).

Perelson, A. S. \& Bell, G. I. (1982). Delivery of lethal hits by cytotoxic $\mathrm{T}$ lymphocytes in multicellular conjugates occurs sequentially but at random times. J. Immunol. 129, 2796-2801.

Perelson, A. S., Macken, C. A., Grimm, E. A., Roos, L. S. \& BonaVIDA, B. (1984). Mechanism of cell-mediated cytotoxicity at the single cell level. V. kinetics of lysis of target cells bound by more than one cytotoxic $\mathrm{T}$ lymphocyte. J. Immunol. 132, 2190-2198.

Perumpanani, A. J., Sherratt, J. A., Norbury, J. \& Byrne, H. (1996). Biological inferences from a mathematical model for malignant invasion. Invasion \& Metastasis 16, 209-221.

PrenN, R. T. (1994). Stimulatory effects of immune reactions upon the growths of untransplanted tumors. Cancer Res. 54, 908-914.

Sampson-Johannes, A. \& CARlino, J. A. (1988). Enhancement of human monocyte tumoricidal activity by recombinant M-CSF. J. Immunol. 141, 3680-3686.

Sherratt, J. A. \& NowAK, M. A. (1992). Oncogenes, anti-oncogenes and the immune response to cancer: A mathematical model. Proc. R. Soc. Lond. B 248, 261-271.

Sherratt, J. A., Sage, E. H. \& Murray, J. D. (1993). Chemical control of eukaryotic cell movement: A new model. J. theor. Biol. 162, 23-40.

Sherratt, J. A., Lewis, M. A. \& Fowler, A. C. (1995). Ecological chaos in the wake of invasion. Proc. Natl. Acad. Sci. U.S.A.92, $2524-2528$

Sozzani, S., Luini, W., Molino, M., Jilek, P., Bottazzi, B. Cerletti, C. ET AL. (1991). The signal transduction pathway involved in the migration induced by a monocyte chemotactic cytokine. J. Immunol. 147, 2215-2221.

TOMLINSON, I. P. M. \& BODMER, W. F. (1995). Failure of programmed cell death and differentiation as causes of tumors: Some simple mathematical models. Proc. Natl. Acad. Sci. U.S.A. 92, 11130-11134.

van Damme, J., Proost, P., Lenaerts, J. P. \& Opdenakker, G. (1992). Structural and functional identification of two human, tumor-derived Monocyte Chemotactic Proteins (MCP-2 and MCP-3) belonging to the chemokine family. J. Exp. Med. 176, $59-65$.

VOLPE, J. (1990). Genetic stability and instability in tumours. In: Molecular Basis of Cancer Genes (Sluyser, M., ed.), pp. 9-23. Chichester: Ellis Harwood.

Walter, S., Bottazzi, B., Govoni, D., Colotta, F. \& Mantovani, A. (1991). Macrophage infiltration and growth of sarcoma clones expressing different amounts of monocyte chemotactic Protein/ JE. Int. J. Cancer 49, 431-435.

Wang, J. M., Colella, S., Allavena, P. \& Mantovani, A. (1987). Chemotactic activity of human recombinant Granulocyte-Macrophage Colony-Stimulating factor. Immunology 60, 439-444. 
Wang, J. M., Griffin, J. D., Rambaldi, A., Chen, Z. G. \& Mantovani, A. (1988). Induction of monocyte migration by recombinant macrophage colony-stimulating factor. J. Immunol. 141, 575-579.

WeINBERG, R. A. (1989). Oncogenes, anti-oncogenes, and the molecular bases of multistep carcinogenesis. Cancer Res. 49, 3713-3721.

WHELDON, T. E. (1975). Mitotic autoregulation of normal and abnormal cells: Alternative mechanisms for the derangement of growth control. J. theor. Biol. 53, 421-433.

YAKOVLEV, A. Y. (1996). Threshold models of tumor recurrence. Mathl. Comp. Modelling 23, 153-164.

\section{APPENDIX}

\section{Non-dimensionalization and Parameter Estimation}

The model given by eqns (1-3) is non-dimensionalized by rescaling time by the normal cell death rate $\delta$; space by $L$, a typical cell length; cell densities by the equilibrium density $N_{e}$; and chemical regulator by $f_{0}$, a typical concentration of $\mathrm{MCP}-1$ in human tumours (taken as $1 \times 10^{-10} \mathrm{M}$ ):

$$
\begin{aligned}
& t^{*}=\delta t, \quad x^{*}=x / L, \quad \quad^{*}=l / N_{e}, \quad m^{*}=m / N_{e}, \\
& n^{*}=n / N_{e}, \quad c^{*}=c / N_{e}, \quad f^{*}=f / f_{0}, \\
& D_{l}^{*}=\frac{D_{l}}{\delta L^{2}}, \quad \chi_{l}^{*}=\frac{\chi_{l} f_{0}}{\delta L^{2}}, \quad D_{m}^{*}=\frac{D_{m}}{\delta L^{2}}, \\
& D_{n}^{*}=\frac{D_{n}}{\delta L^{2}}, \quad D_{f}^{*}=\frac{D_{f}}{\delta L^{2}}, \quad D_{c}^{*}=\frac{D_{c}}{\delta L^{2}}, \\
& \alpha^{*}=\alpha f_{0} / \delta, \quad N^{*}=N / N_{e}, \quad I^{*}=I /\left(N_{e} \delta\right), \\
& \sigma^{*}=f_{0} \sigma, \quad k_{1}^{*}=k_{1} f_{0} N_{e} / \delta, \\
& k_{2}^{*}=k_{2} / \delta, \quad \delta_{l}^{*}=\delta_{l} / \delta, \quad \delta_{f}^{*}=\delta_{f} / \delta, \\
& \delta_{c}^{*}=\delta_{c} / \delta, \quad \beta^{*}=\beta N_{e} /\left(f_{0} \delta\right) .
\end{aligned}
$$

Applying these and omitting the asterisks for notational simplicity gives the dimensionless model:

$$
\begin{aligned}
& \frac{\partial l}{\partial t}=D_{l} \nabla^{2} l-\chi_{l} \nabla(l \nabla f)+\frac{\alpha f(N+1)}{N+l+m+n} \\
& \quad+I(1+\sigma f)-k_{1} f m+k_{2} c-\delta_{l} l \\
& \frac{\partial m}{\partial t}=D_{m} \nabla^{2} m+\frac{\xi m(N+1)}{N+l+m+n}-m-k_{1} f l m \\
& \frac{\partial n}{\partial t}=D_{n} \nabla^{2} n+\frac{n(N+1)}{N+l+m+n}-n \\
& \frac{\partial f}{\partial t}=D_{f} \nabla^{2} f+\beta m-\delta_{f} f \\
& \frac{\partial c}{\partial t}=D_{c} \nabla^{2} c+k_{1} f l m-k_{2} c-\delta_{c} c .
\end{aligned}
$$

There is insufficient experimental data available to determine all the dimensionless model parameters. We list here generic order of magnitude estimates, with brief justifications. All the phenomena described in this paper occur for ranges of parameters within these estimates.

$\alpha-10^{-2}$ : macrophages are a mature form of blood monocyte, which reside in tissues and do not normally proliferate. With certain stimuli, including some tumour-derived chemicals, they may proliferate, but at a low level (Bottazzi et al., 1990), particularly in humans (Mantovani et al., 1992).

$N-10^{1}: N$ is a measure of initial growth rate, and of subsequent response to crowding, and should be of the same order of magnitude as the normal cell death rate.

$I-10^{-2}: I<\delta_{l}$ must hold for the normal tissue steady state to be non-negative. In addition, the proportion of macrophages in normal tissue is relatively small. $\sigma-10^{0}-10^{2}: \quad \sigma$ varies with the response of macrophages to chemoattractants, which will change for different cell lines.

$k_{1}, k_{2}-10^{0}-10^{2}$ : activation, complex formation, and lysis have time scales of hours (Hamilton \& Adams, 1987), and hence a rate one or two orders of magnitude larger than the death rate of normal cells (a typical cell cycle time is of the order of 100 hours). $\delta_{l}-10^{-1}$ : macrophages survive in tissue for weeks or months, compared to a turnover time of days for normal cells, so that macrophage death rate is expected to be at least an order of magnitude smaller than that for normal cells.

$\xi-10^{1}: \xi$ represents the growth advantage of mutant cells, and thus must be greater than one; however mutant cell growth should still be of the same order of magnitude as for normal cells.

$\beta, \delta_{f}-10^{0}-10^{1}$ : The dimensionless chemical production and decay rates should be of the same order of magnitude, and the chemical regulator is expected to decay at a faster rate than normal cells die.

$\delta_{c}-10^{-1}-10^{0}$ : The complex death rate is expected to be of the order of magnitude of the death rates of its component cell types, macrophages $\left(10^{-1}\right)$ and mutant cells $\left(10^{\circ}\right)$.

The coefficients governing random motility of macrophages, mutant cells, normal cells, and complexes, are assumed to be constant, having no dependence on any of the variables. In fact it is to be expected that such coefficients will vary with density, and possibly also the local concentration of regulatory chemicals. In addition the different cell types are assumed to have the same typical order of magnitude of $10^{-15} \mathrm{~m}^{2} \mathrm{~s}^{-1}$ for their random motility coefficients. We need estimates for the normal cell death rate $\delta$ and a typical cell length $L$ in order to nondimensionalise these values. Equation (2b) gives a cell cycle time of $\log 2 / \delta$, and so assuming a normal 
cell cycle time of 100 hours gives $\delta=\log 2 /$ $100 \mathrm{~h}=2 \times 10^{-6} \mathrm{~s}^{-1}$. A typical cell length is $L=10 \mu \mathrm{m}$, and using these figures gives dimensionless coefficients $D_{l}=D_{m}=D_{n}=5 . D_{f}$ is expected to diffuse substantially faster than cells random motion, but since increasing $D_{f}$ leads to a pattern forming bifurcation, we leave it as a free parameter. Concerning the random motion of complexes, it is not clear whether the formation of a complex may render the cells immobile, or at least substantially reduce random movement. Since two cells are bound together in the complex, we halve the value for the random motility coefficient of unbound cells, giving $D_{c}=2.5$. This choice is based purely on our intuition, but in fact the qualitative nature of the solutions does not depend on the particular value, even if it is set to zero. Regarding macrophage chemotaxis, in Section 7 the parameters for random and directed motion of macrophages in response to MCP-1 are estimated from experimental data. 